\title{
DAMAGE RHEOLOGY MODEL AND DECAY LAW OF AFTERSHOCK ACTIVITY
}

\author{
V. Lyakhovsky ${ }^{1}$ and Y. Ben-Zion ${ }^{2}$ \\ ${ }^{1}$ The Geological Survey of Israel, Jerusalem, 95501, Israel \\ ${ }^{2}$ Department of Earth Sciences, University of Southern California Los Angeles, CA, 90089-0740, USA
}

\begin{abstract}
Aftershocks are the response of a damaged rock surrounding large earthquake ruptures to the stress perturbations produced by the large events. Lyakhovsky et al. (1997a) developed a damage rheology model that provides a quantitative treatment for macroscopic effects of evolving distributed fracturing with local crack density represented by a damage state variable. A thermodynamically-based equation for damage evolution accounts for both degradation and healing of rock as a function of on-going deformation. The governing material properties are constrained by analyses of stress-strain and acoustic emission laboratory data during deformation leading to brittle failure of rocks. For analysis of aftershocks, we consider the relaxation process of a material following the application of a strain step associated with the occurrence of a main shock. The coupled differential equations governing the damage evolution and stress relaxation processes are written in non-dimensional form by scaling the elastic stress to its initial value and the time to characteristic time of damage evolution $\tau_{\mathrm{d}}$. With this, the system behavior is controlled by the single non-dimensional ratio $R$ representing the ratio between damage time scale to the Maxwell relaxation time $\left(R=\tau_{\mathrm{d}} / \tau_{\mathrm{M}}\right)$. For very small $R$ there is no relaxation and the response consists of constant elastic strain leading to constant rate of damage increase until failure. For very large $R$ there is rapid relaxation without significant change to the level of damage. For intermediate cases the equations are strongly coupled and nonlinear. The analytical solution for the damage evolution can be fitted well for various values of $R$ with a power law similar to the modified Omori law for aftershocks. This also holds for 3-D numerical simulations of aftershock sequences in a multi-layered lithosphere model. Analytical and numerical results suggest that high values of $R$, corresponding to low viscosity material, produce diffuse aftershock sequences, while low values of $R$, corresponding to more brittle material, produce clear aftershock sequences.
\end{abstract}

\section{INTRODUCTION}

Rocks exhibit a wide variety of rheological behaviors ranging from ductile plastic flow and viscoelastic deformation in the earth mantle and lower crust, to fracture processes controlling the mechanical response and stability of rock mass in the seismogenic zone. A great challenge of theoretical geodynamic studies is to incorporate the interaction between mantle and lower crust into models that simulate deformational processes in the upper crust. Fundamental nonlinear aspects of rock deformation, such as microcrack and flaw nucleation and development of process zones at rupture tips do not have at present accepted quantitative theoretical framework. These aspects are of crucial importance for evolutionary self-organization of faults at various spatio-temporal domains. Lyakhovsky et al. (1997a) developed a thermodynamically-based version of damage rheology, which holds a potential for providing a framework for understanding realistic processes of rock deformation such as those mentioned above. The theoretical results are written in terms of macroscopic variables that can be measured directly in the laboratory. Ben-Zion et al. (1999), Lyakhovsky et al. (2001) and Ben-Zion \& Lyakhovsky (2002) showed that the above damage rheology model can be used to understand deformation associated with large earthquake cycles, evolving fault geometries, frequencysize statistics of earthquakes, accelerated seismic release and more.

In the flowing sections we briefly list some manifestations of distributed damage in natural rocks and rock samples which form the experimental basis for our theoretical damage approach. Then we present the main equations of the damage rheology model. Finally we give a 1-D analytical solution and 3-D numerical simulations that indicate a decay of seismic activity after brittle failures in a form similar to the modified Omori law for aftershocks. 


\section{DISTRIBUTED DAMAGE IN ROCKS}

Pioneering studies of fractures and faults treated the crust as an infinite, perfectly elastic medium (e.g., Anderson, 1951). Subsequent studies accounted for the finite length of faults, and the perturbation to the regional stress field due to the proximity of additional faults (e.g., Chinnery, 1966). Field mapping often shows that the density of faults depends on the scale of the map, so higher resolution increases the number of faults in a given domain (Scholz, 1990). This complexity limits the use of methods that specify the positions of isolated cracks in fault zones. Field observations suggest that the size of the process (damage) zone grow with the size of the fracture, in violation of the premises of the critical stress intensity factor approach (Scholz et el., 1993; Vermilye and Scholz, 1998). This is decisively documented around dikes that form by the injection of magma into fractures (Delaney et al., 1986; Weinberger et al., 1995, 2000; Hoek, 1995; Rubin, 1995).

Damage in the form of microcracks profoundly affects rock strength and rock elastic parameters (Nishihara, 1957; Zoback and Byerlee, 1975; Schock, 1977; Schock and Louis, 1982; Lockner, and Byerlee, 1980; Alm et al., 1985; Reches and Lockner, 1994; Pestman and Munster, 1996) and leads to vanishing elastic moduli at large stresses just before failure (e.g., Lockner and Byerlee, 1980; Lockner et al., 1992; Hamiel et al., 2004). The investigations of fracturing of rocks (Yukutake, 1989; Lockner et al., 1991; Reches and Lockner, 1994) show that this process can not be described in terms of singlecrack propagation. While linear elastic fracture mechanics assumes the size of the inelastic zone at the crack tip to be negligibly small the experiments show that this zone has a significant size. The finite size effect of the fracture process zone is often treated with models which specify a cohesive zone near the crack tip within the plane of the crack (Dugdale, 1960; Barenblatt, 1962, 1996; Ida, 1972; Palmer and Rice, 1973, Rubin, 1993; Willemse and Pollard, 1998). This approach eliminates an unphysical crack-tip singularity and is useful when the crack geometry is well defined. In most engineering and rock-like materials a propagating crack is preceded by an evolving damage zone around its tip (e.g., Bazant and Cedolin, 1991; Lockner et al., 1991), which controls the macro-crack trajectory and growth rate (Huang et al., 1991; Chai, 1993, Zietlow and Labuz, 1998). Thus it is desirable to account explicitly for the distribution of damage in simulations of fault evolution.

\section{THEORY}

We briefly present here the physical basis of continuum damage mechanics. Detailed explanations and comparisons with rock mechanic experiments may be found in Lyakhovsky et al. (1997a) and Hamiel et al. (2004). The damage parameter provides a measure of the effect that distributed microcracks have on the macroscopic response of rocks on applied deformation. A volume-averaged measure of damage represents the density of microcracks at a length scale much larger than the length of a typical crack, yet much smaller than the linear size of the volume considered. In a specimen deformed in a rock-mechanics experiment, microcrack density can be measured over a sub-centimeter scale where it is considered as distributed damage. On the other hand, at the crustal length scale, individual faults shorter than the dominant through-going structure can be considered as distributed damage. For any system with a sufficiently large number of cracks, one can define a representative volume in which the crack density is uniform. Following Kachanov (1986) the damage variable is related to the reduction of the elastic moduli of a spatial domain relative to those of an ideal crack free solid. Rabotnov (1988) related the damage variable to a reduction of the effective cross-section area that supports the load. Fiber-bundle models of damage (Newman \& Phoenix, 2001; Turcotte et al., 2003) share the same idea, where cracks are equivalent to torn fibers.

The present damage rheology model (Lyakhovsky et al., 1997a) treats two aspects of the physics of damage: (1) A mechanical aspect - sensitivity of the macroscopic elastic shear modulus to distributed cracks and to the sense of loading, and (2) a kinetic aspect - the evolution of damage (degradation/recovery of elasticity) in response to loading. The cumulative effect of distributed micro- 
cracks and flaws in the elastic material leads to non-linearity, which is described by an energy potential equation of the form:

$$
U=\frac{1}{\rho}\left(\frac{\lambda}{2} I_{1}^{2}+\mu I_{2}-\gamma I_{1} \sqrt{I_{2}}\right) .
$$

where $I_{1}=\varepsilon_{k k}$ and $I_{2}=\varepsilon_{i j} \varepsilon_{i j}$ are two strain invariants of the strain tensor $\varepsilon_{\mathrm{ij}}$. This is the simplest mathematical expression for the elastic energy that expands the classical form with two Lame constants $\lambda$ and $\mu$, being a non-analytical second order function of two strain invariants $I_{1}$ and $I_{2}$ and additional modulus $\gamma$. Lyakhovsky et al. (1997b) show that non-linear elastic deformation of rocks including dilation is fitted well with stress-strain relations derived from the potential (1). In opposite to the Murnaghan (1951) expression, who added a higher order term to the elastic energy, the present approach preserves its non-linear features even for small deformation.

The kinetic aspect of the damage rheology model is introduced by making the Lame parameters $\lambda, \mu$ and the additional modulus $\gamma$ functions of the damage parameter $0 \leq \alpha \leq 1$. The amount of damage $\alpha$ evolves in time as a result of an applied load. Using the balance equations of the energy and entropy, and accounting for irreversible changes related to viscous deformation and material damage, the equation of damage evolution has the form (Lyakhovsky et al., 1997a)

$$
\frac{d \alpha}{d t}=-C \frac{\partial U}{\partial \alpha},
$$

where the positive constant or function of state variables $C$ provides the non-negative local entropy production related to damage evolution. This approach describes not only damage increase, but also a process of material recovery associated with healing of microcracks, which is favored by high confining pressure, low shear stress and high temperature. Agnon and Lyakhovsky (1995) chose the moduli $\mu$ and $\gamma$ to be linear functions of $\alpha$ and modulus $\lambda$ be constant. Later analysis of laboratory acoustic emission and stress-strain data (Hamiel et al., 2004) show that the quality of data fitting may be improved assuming power law relation between damage and elastic moduli. However, a linear relation is still very reasonable approximation for conditions in the seismogenic zone. Increasing the third modulus $\gamma$ from zero for damage free Hookean material to its maximum value at critical damage amplifies the non-linearity of rock elasticity with damage accumulation. Using the elastic energy (1) the equation for kinetic of damage accumulation is (Lyakhovsky et al., 1997a):

$$
\frac{d \alpha}{d t}=C_{d} I_{2}\left(\xi-\xi_{0}\right) \quad \text { for } \quad \xi \geq \xi_{0}
$$

where $\xi=I_{1} / \sqrt{I_{2}}$ is the strain invariant ratio. Equation (3) includes a parameter $\xi_{0}$ that indicates the transition stage from strengthening to degradation. Agnon and Lyakhovsky (1995) and Lyakhovsky et al. (1997a) related this parameter to the angle of internal friction by considering the critical shear stress for Mohr-Coulomb sliding. They received $\xi_{0}=-0.8$ for typical ratios of elastic moduli for damage free material $\lambda / \mu \sim 1$ (Poisson's ratio of 0.25 ) and internal friction angle $\sim 40^{\circ}$ (Eq. 37 and Fig.3 of Lyakhovsky et al., 1997a). This value varies little for different rocks with Poisson ratios between 0.2 and 0.3 and is used for the following up numerical simulations. The parameter $C_{d}$ is the damage rate constant for positive damage evolution (degradation), which defines the time needed to achieve failure after the onset of damage at $\xi=\xi_{0} . \quad C_{d}$ is assumed to be a material property and its value has been estimated to vary from 0.5 to $5 \mathrm{~s}^{-1}$ for different rocks tested at more than $20 \mathrm{MPa}$ confining pressure and room temperature (Lyakhovsky et al., 1997a). Detailed recent analysis of laboratory data (Hamiel et al., 2004) also shows that with the onset of acoustic emission and damage evolution, there is non-reversible deformation before the final macroscopic failure. This can be accounted for using a damage-related viscosity $\eta$, which for positive rate of damage is 


$$
\eta=\frac{\eta_{0}}{\dot{\alpha}}
$$

The viscosity value increases with decreasing damage rate and is infinity large (no any irreversible deformation) for negative rate of damage (healing). The damage-related viscosity accounts for irreversible deformations at the length scale below the length scale of a representative volume for which the average damage parameter was defined.

\section{DECAY OF ACTIVITY (ANALYTICAL SOLUTION)}

The fully 3-D model formulation is significantly simplified in a one-dimensional case. In spite of the non-linearity of the general stress-strain relations based on the elastic potential (1), a corresponding 1D stress-strain still has a linear form

$$
\sigma=2 \mu_{0}(1-\alpha) \varepsilon
$$

with the damage parameter $\alpha$ evolving according to the 1-D version of the damage rate equation (2)

$$
\dot{\alpha}=C_{d} \varepsilon^{2}
$$

Equations $(5,6)$ describe evolving elastic strain and damage as a result of ongoing deformation. Equation (6) differs from the 3-D equation (3) by a factor $\left(\xi-\xi_{0}\right)$, which relates the rate of damage increase with the 3-D structure of the deformation. Viscous relaxation gradually reduces the deviatoric component of the elastic strain leading to decreasing the $\xi$ value. When the factor $\left(\xi-\xi_{0}\right)$ approaches zero, the rate of damage evolution decreases and the system achieves a static state. The fully coupled evolution is simulated numerically and discussed in the next section. Here we assume that the factor $\left(\xi-\xi_{0}\right)$ is of the order of one, which allows analytical analysis of the system behavior. The irreversible strain component before the final macroscopic failure is given by

$$
\sigma=2 \eta e
$$

where $e$ is the rate of gradual irreversible strain and the viscosity $\eta$ is defined in equation (4). The set of equations (4-7) governs the 1-D behavior of the system. A similar set of equations with constant viscosity, excluding eq. 4, was previously analyzed by Lyakhovsky et al. (1997a) to describe a stickslip motion under a constant rate of applied deformation. The solution of equations $(5,6)$ without irreversible strain accumulation prior to the final brittle failure gives a power-law time-to-failure relation consistent with the power-law seismic activation prior to some large earthquakes (Ben-Zion and Lyakhovsky, 2002; Turcotte et al., 2003). The analytical power-law scaling was obtained for a constant applied stress that mimics natural boundary conditions for a system at the stage of accelerating seismic release. This result was confirmed by numerical simulations in a model of rheologically layered lithosphere governed by damage rheology (Ben-Zion and Lyakhovsky, 2002). Here we analyze the system behavior with constant total strain boundary condition. Such a condition is appropriate for a region where aftershock series are collected after a strong event.

We scale the elastic strain to its initial value $\varepsilon_{0}$ and time to the damage time scale $\tau_{d}=1 / C_{d} \varepsilon_{0}^{2}$. The analysis indicates that the system behavior is defined by only one parameter $R$, the ratio between $\tau_{\mathrm{d}}$ and Maxwell time-scale for viscous relaxation $\tau_{\mathrm{M}}$. The latter is

and the ratio $R$ is

$$
\tau_{M}=\frac{\eta}{\mu} \cong \frac{\eta_{0}}{\mu_{0} \dot{\alpha}} \cong \frac{\eta_{0}}{\mu_{0} C_{d} \varepsilon_{o}^{2}}
$$

$$
R=\frac{\tau_{d}}{\tau_{M}} \cong \frac{\mu_{0}}{\eta_{0}}
$$

After elimination the elastic strain and integration, the damage evolution is described by the equation 


$$
\operatorname{erf}\left(\sqrt{\frac{R}{2}}\right)-\operatorname{erf}\left(\sqrt{\frac{R}{2}}(1-\alpha)\right)=\varepsilon_{0}^{2} \exp \left(-\frac{R}{2}\right) \sqrt{\frac{2 R}{\pi}} \cdot t
$$

The solution (10) shows that the system does not follow a power-law scaling. However for a given $R$ value, the calculated rate of damage can roughly be approximated by a power-law function similar to that used for the Omori law. The solution (10) can also be used to estimate the time needed for $\alpha$ to grow from 0 to 1 , which roughly increases as $\exp (R)$. Thus, we expect a faster decay of the fracture process corresponding to shorter aftershock series for small $R$-values, and long-lasting series or even swarms of events for high $R$-values.

\section{DECAY OF ACTIVITY (3-D NUMERICAL SIMULATIONS)}

We present results of 3-D numerical simulations of the coupled evolution of earthquakes and faults in a model consisting of a seismogenic upper crust governed by damage rheology over a viscoelastic layered substrate. Ductile flow in the lower crust and upper mantle is governed by power-law temperature-dependent rheology. Our three dimensional numerical simulations use the Fast Lagrangian Analysis of Continua (FLAC) algorithm (Cundall and Board, 1988; Cundall, 1989; Poliakov et al., 1993). The formulation is explicit-in-time, using an updated Lagrangian scheme to provide the capability for large strains. The FLAC algorithm offers advantages over conventional finite element schemes in case where material instability occurs. Physical instability is modeled without numerical instability if inertial terms are included in the equilibrium equations.

The simulated aftershock statistics depend on the parameter $R$ responsible for the stress relaxation in the seismogenic zone and chosen temperature gradient. The results indicate that model realizations with low $R$-values and low temperature gradient (relatively cold lower crust) has clear aftershock record. In contrast, stress relaxation is very fast in model realizations with high $R$-values and hot lower crust, producing diffuse swarm-like patterns. The analyses of the synthetic aftershock catalogs show that the temporal aftershock distribution can be fitted well for various values of $R$ with a power law similar to the modified Omori law.

\section{REFERENCES}

Agnon, A. \& Lyakhovsky, V., 1995. Damage distribution and localization during dyke intrusion. In: The physics and Chemistry of Dykes. Eds. Baer, G. \& Heimann A., pp. 65-78, Balkema. Brookfield.

Alm, O., Jaktlund, L.L. \& Kou, S. 1985. The influence of microcrack density on the elastic and fracture mechanical properties of Stripa granite. Phys. Earth. Planet. Inter., 40, 161-179.

Anderson, E.M., The dynamics of faulting, 183 pp., Oliver and Boyd, London, 1951.

Barenblatt, G.I., 1962. Mathematical theory of equilibrium cracks in brittle fracture. Acv. Appl. Mech., 7, 55-129.

Barenblatt, G.I., 1996. Scaling, self-similarity, and intermediate asymptotics, Cambridge Univ. Press.

Bazant, Z.P. \& Cedolin, L. 1991. Stability of structures. Elastic, Inelastic, fracture and damage theories, Oxford University Press.

Ben-Zion, Y. \& Lyakhovsky, V., 2002. Accelerating seismic release and related aspects of seismicity patterns on earthquake faults. Pure and Appl. Geophys. 159, 2385-2412

Ben-Zion, Y., Dahmen, K., Lyakhovsky, V., Ertas, D., \& Agnon, A., 1999. Self-driven mode switching of earthquake activity on a fault system. Earth Planet. Sci. Lett. 172,11-21.

Chai, H., 1993. Observation of deformation and damage at the tip of cracks in adhesive bonds loaded in shear and assessment of a criterion for fracture. Int. J. Fracture, 60, 311-326.

Chinnery, M.A., 1966. Secondary faulting. Canad. J. Earth Sci., 3, 163-190,

Cundall, P. A. 1989. Numerical experiments on localization in frictional materials. Ign. Arch., 59:148-159.

Cundall, P. A., \& Board, M.1988. A microcomputer program for modeling large-strain plasticity problems. Numerical Methods in Geomechanics, Procedings of 6th International Conference on Numerical Methods in Geomechanics, Innsbruck, edited by C. Swoboda, pp. 2101-2108, R.A. Balkhema, Brookfield, Vt.

Delaney, P.T., D.D. Polard, J.I. Ziony, and E.H. McKee, 1986. Field relations between dikes and joints: emplacement processes and paleostress analysis. J. Geophys. Res., 91, 4920-4938.

Dugdale, D.S., 1960. Yielding of steel sheets containing slits. J. Mech. Phys. Solids, 8, 100-104.

Hamiel, Y., Liu, Y., Lyakhovsky, V., Ben-Zion, Y. \& Lockner, D., 2004 (in press). A visco-elastic damage model with application to stable and unstable fracturing. Geophys. J. Int. 
Hoek, J.D., 1995. Dyke propagation and arrest in Proterozoic tholeitic dyke swarms, Vestfold Hills, East Antarctica, in The Physics and Chemistry of Dykes, eds. Baer \& Heimann, Balkema, Rotterdam, 79-95, .

Huang, W-L., Kunin, B. \& Chudnovsky, A. 1991. Kinematics of damage zone accompanying curved crack. Int. J. Fracture. 50, 143-152.

Ida, Y., 1972. Cohesive force across the tip of longitudinal shear crack and Griffith's specific surface energy, J. Geophys. Res., 77, 3796-3805.

Kachanov, L.M., 1986. Introduction to continuum damage mechanics, pp. 135, Martinus Nijhoff Publishers.

Krajcinovic, D., 1996. Damage Mechanics. Elsevier, Amsterdam.

Lockner, D.A. \& Byerlee, J.D. 1980. Development of fracture planes during creep in granite. $2^{\text {nd }}$ Conference on Acoustic Emission/Microseismic Activity in Geological Structures and Materials, Trans-Tech. Publications, Clausthal-Zellerfeld, Germany.

Lockner, D.A., Byerlee, J.D., Kuksenko, V., Ponomarev, A. \& Sidorin, A. 1991. Quasi-static fault growth and shear fracture energy in granite. Nature, 350, 39-42.

Lockner, D.A., Byerlee, J.D., Kuksenko, V., Ponomarev, A. \& Sidorin, A. 1992. Observations of quasi-static fault growth from acoustic emission. Fault mechanics and transport properties of rocks, International geophysics series, v. 51, B.Evans and T.-F. Wong (eds.), Academic Press, 3-31.

Lyakhovsky, V., Ben-Zion, Y. \& Agnon, A., 1997a. Distributed damage, faulting and friction. J. Geophys. Res. 102, 27,63527,649 .

Lyakhovsky, V., Ben-Zion, Y.\& Agnon, A., 2001. Earthquake cycle, fault zones and seismicity pattern s in a rheologically layered lithosphere. J. Geophys. Res. 106, 4,103-4,120.

Lyakhovsky, V., Reches, Z., Weinberger, R. \& Scott, T.E., 1997b. Non-linear elastic behavior of damaged rocks, Geophys. J. Int., 130, 157-166.

Lyakhovsky, V.A. \& Myasnikov, V.P., 1985. On the behavior of visco-elastic cracked solid. Physics of Solid Earth, 4, 28-35.

Murnaghan, F.D., 1951. Finite deformation of an elastic solid, John Wiley, Chapman, New York.

Newman W.I. \& Phoenix S.L., 2001. Time-dependent fiber bundles with local load sharing. Physical Rev. E. 63 (2).

Nishihara, M., 1957. Stress-strain relation of rocks, Doshisha Eng. Rev., 8, 32-54.

Palmer, A. C. and Rice, J.R. 1973. The growth of slip surfaces in the progressive failure of over-consolidated clay. Proc. Roy. Soc. London, Ser. A, 332, 527-548.

Pestman, B.J. \& Munster, J.G. 1996. An acoustic emission study of damage development and stress - memory effects in sandstone". Int. J. Rock Mech. Min. Sci., 33, 585-593.

Poliakov A., Cundall P., Podladchikov Yu., Lyakhovsky V. 1993. An explicit inertial method for the simulation of viscoelastic flow: An evaluation of elastic effects on diapiric flow in two and three layer models. Proceeding of NATO Advanced Study Institute on Dynamic Modeling and Flow in the Earth and Planets. In: D.B.Stone and S.K.Runcorn (eds.), Flow and Creep in the Solar system: Observations, Modeling and Theory, Kluwerr, Holland, 175-195.

Rabotnov, Y.N., 1988. Mechanics of deformable solids, pp. 712, Moscow, Science.

Reches, Z. \& Lockner, D.A., 1994. Nucleation and growth of faults in brittle rocks, J. Geophys. Res., 99, 18159-18173.

Rubin, A.M., 1995. Propagation of magma filled cracks. Ann. Rev. Earth Planet. Sci. 8, 287-336.

Rubin, A.M. 1993. Tensile fracture of rock at high confining pressure: Implications for dike propagation. J. Geophys. Res. 98, $15,919-15,935$.

Schock, R.N. \& Louis, H. 1982. Strain behavior of a granite and a graywacke sandstone in tension. J. Geophys. Res., 87, 78177823.

Schock, R.N. 1977. The response of rocks to large stresses. Impact and explosion cratering D.L. Roddy, R.O. Pepin, and R.B. Merrill (editors), Pergamon press, New York, NY.

Scholz, C. H., Dawers, N. H., Yu, J.-Z., Anders, M. H. \& Cowie, P.A., 1993. Fault growth and fault scaling laws: Preliminary results, J. Geophys. Res., 98, 21951-21961.

Scholz, C.H., 1990. The mechanics of earthquakes and faulting, pp. 439, Cambridge University, England.

Turcotte, D.L., Newman, W.I. \& Shcherbakov, R., 2003. Micro and macroscopic models of rock fracture. Geophys. J. Int. 152, 3 : $718-728$.

Vermilye, J.M. \& Scholz, C.H., 1998. The process zone: A microstructural view of fault growth, J. Geophys. Res., 103, 12,22312,236 .

Weinberger, R., Baer, G., Shamir, G. \& Agnon, A., 1995. Deformation bands associated with dyke propagation in porous sandstone, Makhtesh Ramon, Israel, in Physics and Chemistry of Dykes, eds. Baer \& Heimann, 95-112, Balkema, Rotterdam.

Weinberger, R., Lyakhovsky, V., Baer, G. \& Agnon, A. 2000. Damage zone around en-echelon dyke segments in porous sandstone. J. Geophys. Res. 105:3115-3133.

Willemse, E.J.M. \& Pollard, D.D. 1998. On the orientation and patterns of wing cracks and solution surfaces at the tips of a sliding flaw or fault. J. Geophys. Res., 103, 2427-2438.

Yukutake, H., Fracturing process of granite inferred from measurements of spatial and temporal variations in velocity during triaxial deformation, J. Geophys. Res., 94, 15639-15651, 1989.

Zietlow, W.K. \& Labuz, J.F. 1998. Measurements of the intrinsic process zone in rock using acoustic emission". Int. J. Rock Mech. Min. Sci., 35, 291-299.

Zoback, M.D. \& Byerlee J.D. 1975. The effect of mickrocrack dilatancy on the permeability of Westerly granite. J. Geophys. Res., 80-B, 752-755 